\title{
Attrition and Cryogenic milling powder production for Low Pressure Cold Gas Spray and composite coatings characterization
}

\author{
M. Robotti, S. Dosta*, I.G. Cano, A. Concustell, N. Cinca, J.M. Guilemany \\ CPT Thermal Spray Centre, Departament d'Enginyeria Química I Metal-lúrgica, Universitat de Barcelona, Martí i Franquès 1, E-08028 Barcelona, Spain
}

\section{A R T I C L E I N F O}

Article history:

Received 22 January 2016

Received in revised form 8 April 2016

Accepted 19 April 2016

Available online $\mathrm{xxxx}$

\section{Keywords:}

Nano particles

Particle-reinforced composites

Functional composites

Low Pressure Cold Gas Spray

Coatings

\begin{abstract}
A B S T R A C T
Inside the wide field of thermal spray, the possibility to spray ceramic nano-particles by Low Pressure Cold Gas Spray (LP-CGS) represents an interesting and innovative trend. In this work, titanium dioxide $\mathrm{TiO}_{2}$ nanoparticles were mixed with a polymer in order to obtain a tailor-made nanocomposite powder and afterwards cold-sprayed coatings were produced, which might be attractive for their photocatalytic activity. Firstly, two different routes were used to incorporate the ceramic nanoparticles within the polymeric matrix: Attrition Milling (AM) and Cryogenic Milling (CM). Samples composition was not varied, while milling time was changed. The main objective was to investigate the mechanical physical union of $\mathrm{TiO}_{2}$ nanoparticles around polymeric microparticles. The effect of ceramic particles on the structure and morphology of the polymer, as well as the influence of the temperature of the different combining processes were studied. The fundamental properties of the nanocomposite mixture were investigated by Scanning Electron Microscopy (SEM), Laser Scattering (LS Beckman Coulter) and X-ray Diffraction (XRD). Secondly, the best mechanically combined mixture was selected in order to be sprayed by LPCGS technology. Adequate spraying parameters were chosen in order to develop different composite coatings. Thickness, roughness and porosity of manufactured products were measured.
\end{abstract}

(c) 2016 The Society of Powder Technology Japan. Published by Elsevier B.V. and The Society of Powder Technology Japan. All rights reserved.

\section{Introduction}

Nanocomposites materials are nowadays in the spotlight of both industry and research groups. The increasing interest in this type of composites arises from the possibility of preparing tailormade materials that combine the advantages of ceramic nanoparticles, for instance electrical conductivity and photocatalysis [1,2], with the ones of polymers, e.g. ductility, deformability and plasticity. Nanostructured ceramics are conventionally defined as inorganic materials composed of structural units with a size scale of less than $100 \mathrm{~nm}$ in any dimension. Nanoceramics are classified as zero-dimensional nanocrystals, one-dimensional nanowires and nanotubes, two-dimensional nanofilms and nanowalls, and three-dimensional bulk materials with at least one nanocrystalline phase. These materials permit to obtain superior and unique properties in conventional ceramics with coarser structured units. As a large fraction of interfacial areas is created between a nanofiller and a polymeric matrix due to the high surface to volume ratio of nanoparticles, it is expected that the interphase formed in nanocomposites will have more importance for the final properties

\footnotetext{
* Corresponding author. Tel.: +34 934034449

E-mail address: sdosta@cptub.eu (S. Dosta).
}

than that in conventional polymer composites. However, this only should occur with uniform dispersion of nanoparticles [3,4], which most of the times is a really difficult challenge. For this reason, characterization of interphases in nanocomposites with uniform dispersion is a key factor to understand their final properties and performance. Up to this moment, several strategies $[5,6]$ have been followed to achieve uniform nanoparticle dispersions within polymer matrices; however, most of them required the use of solvents, chemical modification of the matrix and/or the filler, long processing times, and even sometimes high processing temperatures. Nevertheless, they do not seem to ensure uniform dispersion in terms of isolated nanoparticles when sizes of particles are less than $50 \mathrm{~nm}$ and filler loads are greater than $5 \%$ by weight (wt). Recently, with solid-state methods, such as Attrition milling (AM) $[7,8]$, mechanical mixture of nanoparticles that remain well-bonded around the polymer has been achieved. Usually, AM provides good results with small chemical modifications, if any, of the polymer or the nanoparticles, avoiding, at the same time, the use of solvents and high processing temperatures [9]. A second mixing method, Cryoattrition Milling CM, commonly used for amorphous alloys and intermetallic materials, is also taken into account as new potential alternative for composite powder blending at low energy conditions. The main theory of the dispersing mechanism of 
particles on polymer proposed in this paper is the so-called mechanical anchoring $[3,10]$. This phenomenon takes into account the deformation of the polymer, especially on the surface, and the consequently mechanical adhesion of ceramic particles onto it. This approach has been studied in recent years and considerable effort has been devoted to the development of methods for the preparation of composite particles consisting of polymer cores covered with shells of different chemical composition. Several of these composites have been used as catalysts, coatings, raw materials recovery, drug delivery and anticorrosion protection $[1,11,12]$. In this case, ceramic-polymeric nanocomposites will be manufactured and developed for coating production through the Low Pressure Cold Gas Spray technology [22,23,26]. Polymeric particles with shells of nanoceramics do not lose their flowability and this is a significant aim inside the spraying process [24]. Therefore, the present work is twofold: (i) polymer nanocomposite obtainment by Attrition milling and Cryogenic milling of a polymer with oxide nanoparticles and (ii) coating build up by LP-CGS with the optimized powders.

\section{Experimental materials and methods}

In this work, titanium dioxide $\mathrm{TiO}_{2}$ nanoparticles were room temperature and cryogenically milled with a copolymer as ductile matrix. The polymeric material is a commercial one: Halar ${ }^{\circledR} 6014$ from Solvay. Laser Scattering (LS 13 320, Universal Liquid Module) was carried out in order to determine the particle size distribution of the raw materials. Two different mixing systems were used to produce composite powder materials: Attrition Mill (Model 01HD, Union Process Inc.) and Cryogenic Mill (Attritor 01HD, Union Process Inc.). Top surface of starting powders and milled composite mixtures as well as cross sections of final coatings were observed by SEM scanning electron microscope (Pro-X, Phenom). Diffraction data were studied by X'Pert PRO MPD diffractometer (PANalytical). Composite coatings were obtained by means of Low Pressure Cold Gas Spray system (Dymet 423). Produced coatings were mounted in an epoxy resin (EpoFix, Struers) and subsequently metallographic preparation was carried out.

\subsection{Polymer}

The polymeric matrix is a semi-crystalline, melt processable resin copolymer. The polymeric chain consists in a sequence of ethylene and chlorotrifluoroethylene monomers. Good chemical resistance, excellent thermal properties, optimal permeation resistance, anti-corrosion behaviour and outstanding flame resistance are just part of its interesting characteristics. Since the aim of this manuscript was to study the behaviour of polymeric and ceramic nanoparticles inside both AM and CM systems, it was necessary to study a narrow range of particle size distribution in order to comprehend well the mixing mechanisms. For this reason, the polymer was sieved in two different ranges: 20-40 $\mu \mathrm{m}$ (PR101S1 in Fig. 2a) and 60-80 $\mu \mathrm{m}$ (PR101-S2 Fig. 2b). Though this way, it is possible to work with more homogeneous starting materials, so that it is easier to understand what happens to finest particles and to biggest ones during the mixing processes: also, the particle size of the final composite powder will be important regarding cold spray application. Not only the deformation of polymeric particles is important, but also the crystallinity of the material; in Fig. 3 the XRD pattern is shown and it is possible to notice a crystalline diffraction peak at $17.4^{\circ}$ and a second broader peak at around $40^{\circ}$ which represents the amorphous part of the polymeric matrix.

\subsection{Titanium dioxide}

LS curves showed in Fig. 4a confirm the fact that the titania powder possesses a medium particle size distribution of $1.88 \mu \mathrm{m}$. Two peaks can be observed: a first peak consists in submicrometric particles from $0.05 \mu \mathrm{m}$ to $0.3 \mu \mathrm{m}$, while the second peak represents the majority of material volume. In this second case $\mathrm{d}_{90}$ corresponds to $4.4 \mu \mathrm{m}$. In the SEM top surface micrograph on Fig. $4 \mathrm{~b}$ it is possible to notice both small particles and groups of agglomerated ones. This phenomenon is mainly due to electrostatic agglomeration because of the small dimensions of titania. Nevertheless, powders with these grain sizes could not be easily sprayed by Low Pressure Cold Gas Spray technique [28] due to their bad flowability. Diffraction peaks of $\mathrm{TiO}_{2}$ shown in Fig. 5 correspond to the anatase phase and possess a wide width due to the nanometric size of the powder. This broadening is an intrinsic characteristic of the material, i.e. the large width is due to the nano sized crystals of the material. Titania nano dimensions were specifically selected in order to achieve the best mechanical union with micrometric polymeric particles. The new composite material has a polymeric matrix surrounded by $\mathrm{TiO}_{2}$ nanoparticles and this way it is possible to have a correct flowability inside the LP-CGS nozzle and also be able to spray ceramic nanomaterials $[18,21,25]$. (a)

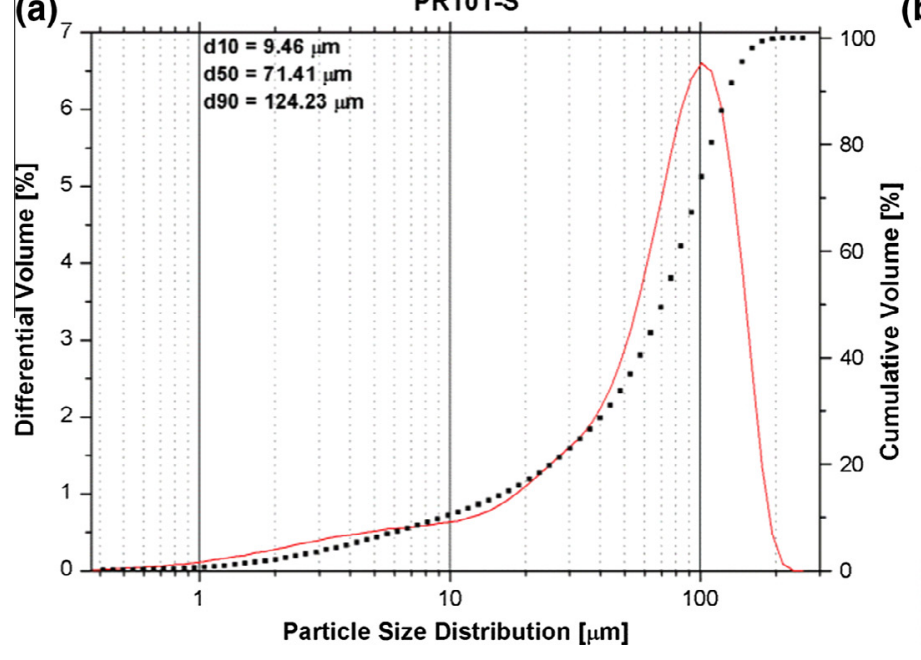

(b)

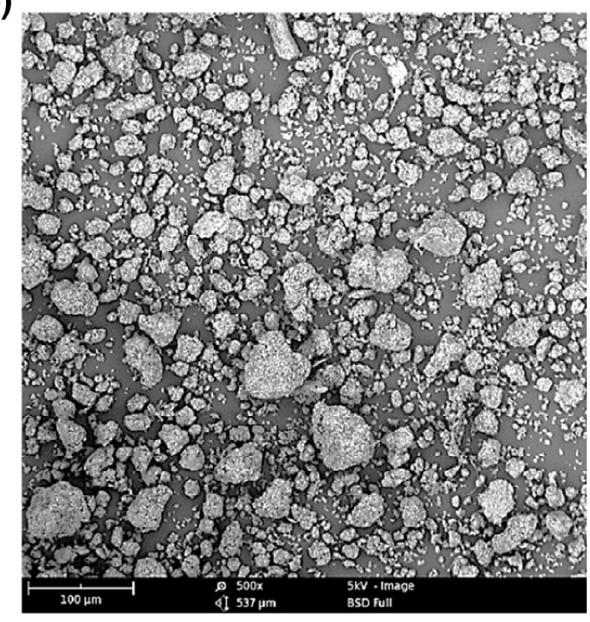

Fig. 1. Wide particle size distribution (a) and SEM (secondary electrons) top surface (b) of polymer. 
(a)

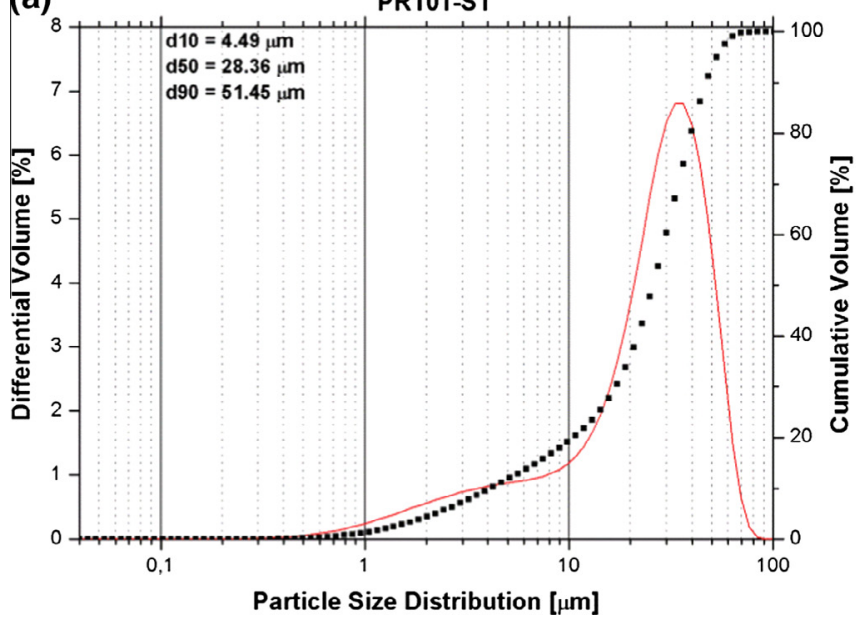

(b)

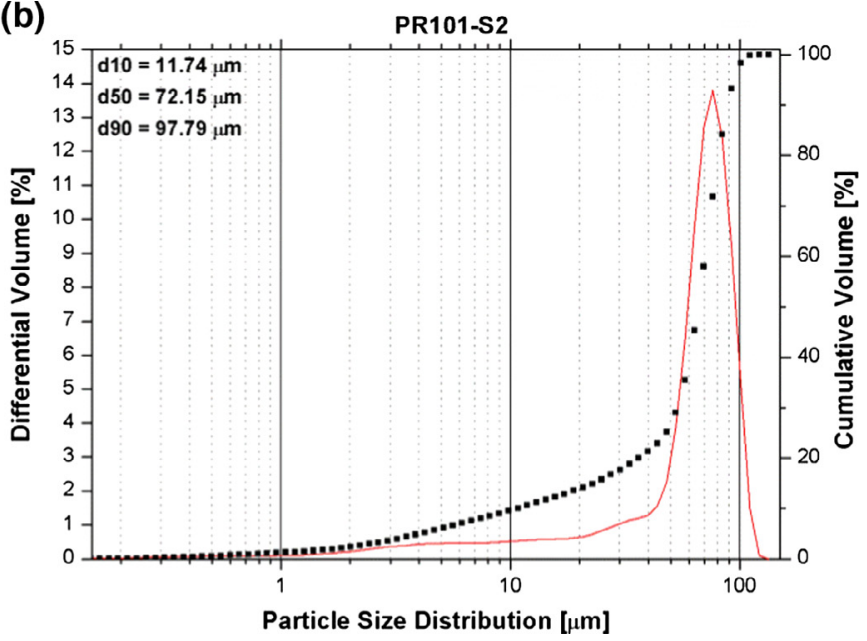

Fig. 2. LS of sieved polymeric particles: PR101-S1 (a) and PR101-S2 (b).

PR101-S

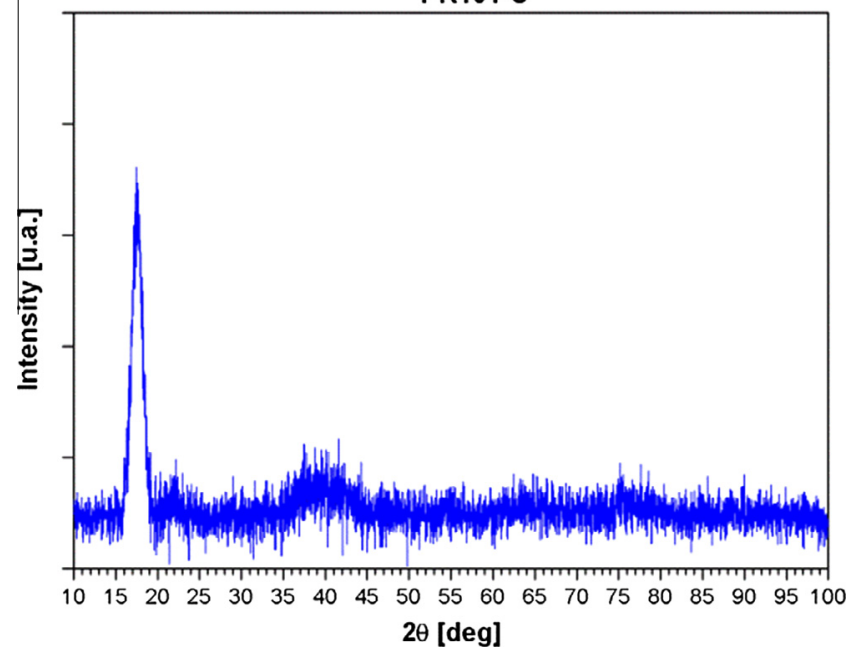

Fig. 3. XRD analysis of the polymeric material.

(a)

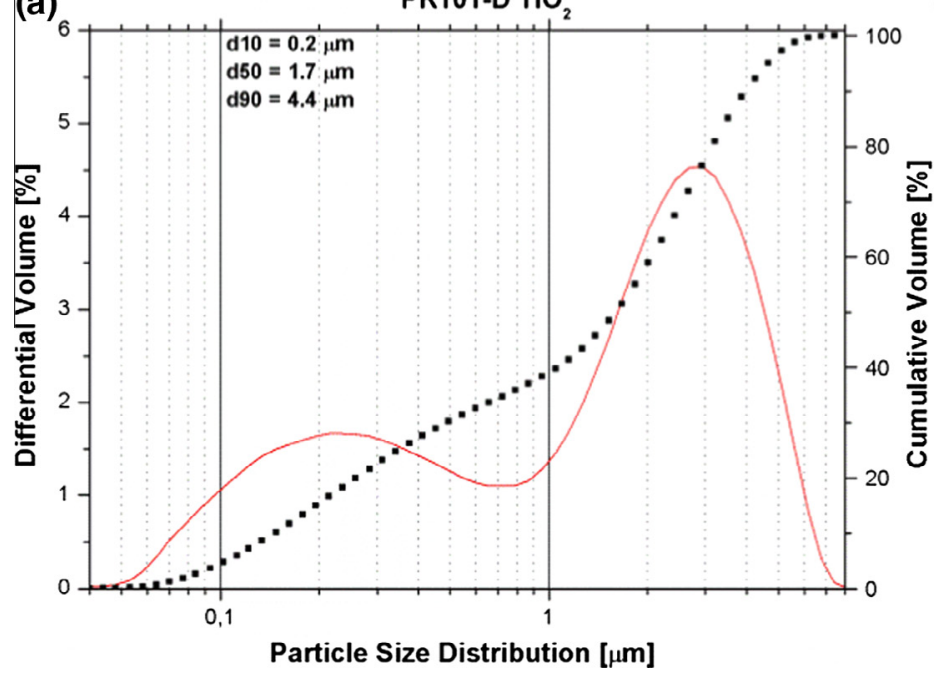

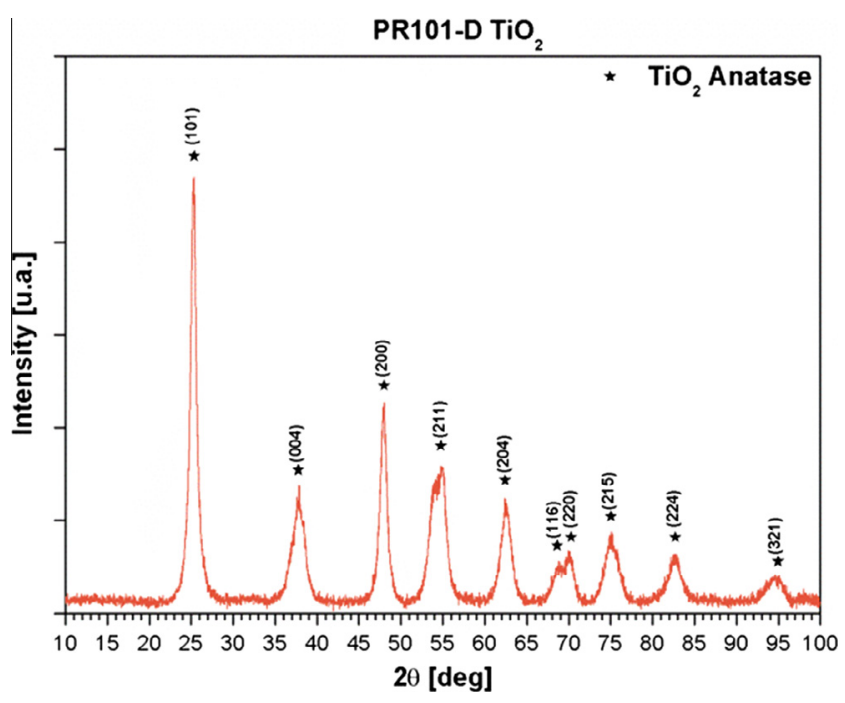

Fig. 5. XRD anatase pattern of titanium dioxide.

Fig. 4. LS analysis (a) and SEM (secondary electrons) top surface of $\mathrm{TiO}_{2}$ powder (b). 


\subsection{Mechanical milling techniques}

Mechanical milling techniques are typically solid-state powder processes where cold welding and fracturing processes take commonly place [30]. They are typically used for production of crystalline, stable and metastable materials, amorphous alloys, intermetallic compounds and, in general, to develop homogeneous materials in form of High Energetic Conditions (HEC) are often used in order to achieve high impact energies that can plastically deform or fracture the material in powder form [15-17]. Strong impacts among balls, powder, and walls of containers can significantly reduce particle size distribution. Vessels and impellers of ceramic, metallic or polymeric materials can be used in function of their hardness and in function of the materials of the powders. In this work, typical high energetic conditions of crushing and reducing particles sizes are not applied. Low Energetic Conditions (LEC) are applied in order to mix properly ceramic-polymeric nanocomposite materials and study the incrustation of ceramic nanoparticles around the bigger polymeric ones. The hardness of these two different materials was taken into account during the selection because a ductile soft polymeric was selected as matrix in which hard ceramic nanoparticles can be incrusted and mechanically adhered thanks to the kinetic energy transferred from ceramic balls to the material. Two mechanical techniques are used in this study case: Attrition and Cryogenic Milling. These different mechanisms allow study the behaviour of the particles and their final characteristics which have to be the best ones for the following LP-CGS spraying process [24].

\subsubsection{Attrition Milling (AM)}

AM consists in a vertical container filled with balls and with inside an impeller. A powerful motor rotates the impeller, which in turn rotates the balls in the container. The higher the revolutions per minute (rpm) are, the higher the impact energies between the materials. This way the temperature can increase and fractures and micro-welding mechanisms can happen. For this reason, water cool system is coupled.

\subsubsection{Cryogenic Milling ( $C M)$}

$\mathrm{CM}$ possesses the same configuration of $\mathrm{AM}$, but with a different atmosphere; instead of air this equipment works with liquid nitrogen medium. For this reason the system works below the ductilebrittle transition temperature of the polymer [3,5]. The use of $\mathrm{N}_{2}$ is useful to avoid chemical reactions and to work in a specific range of temperatures in which the material is a fragile state [31]. Inside the vessel, the milling environment consists of milling balls, powders and flowing liquid nitrogen. Usually, CM is used to strengthen materials through grain size refinement and the dispersion of fine, nano-meter scale particles; in this scientific work it is interesting to observe the embrittlement effect of the polymeric particles and their reduction in dimensions. Another common difference among $\mathrm{AM}$ and $\mathrm{CM}$ is that the liquid nitrogen present in $\mathrm{CM}$ allows much shorter milling times to reach the finest particle sizes and smaller recrystallized grains when compared to mechanical alloying performed in air [14]. In the following Table 1, milling parameters which were used in this research are shown.

Table 1

Mixing parameters.

\begin{tabular}{lll}
\hline & Cryogenic milling & Attrition milling \\
\hline Milling time $(\mathrm{h})$ & $0.5 ; 1 ; 4$ & $0.5 ; 1 ; 4$ \\
Impeller material & Steel & Steel \\
Vessel material & Steel & Steel \\
Grinding medium & $\mathrm{N}_{2}$ & Air \\
Milling Temperature & $<-76{ }^{\circ} \mathrm{C}$ & $20{ }^{\circ} \mathrm{C}$ \\
Balls material & $\mathrm{Al}_{2} \mathrm{O}_{3}$ & $\mathrm{Al}_{2} \mathrm{O}_{3}$ \\
\hline
\end{tabular}

Composition of the starting powders, mixing time, ball-tomaterial ratio, material balls and containers are kept constant. The objective is to study the mechanical anchoring of the two different materials only changing temperature and atmosphere inside the mixing volumes. The grinding medium influences directly the milling efficiency. AM works at environment temperature $\left(25^{\circ} \mathrm{C}\right)$, controlled by water cooling system, while $\mathrm{CM}$ works at cryogenic temperatures, i.e. temperatures below $-76^{\circ} \mathrm{C}$, which represents the brittle-ductile temperature of the polymer.

\section{Results and discussion}

Firstly, characterization of initial materials was done. Polymeric and ceramic powders were studied by different experimental techniques. Secondly, a deeper characterization of final coatings is shown. The most interesting properties of these composite products are exposed and interesting future applications are proposed as well.

\subsection{Characterization of raw materials}

In this first section, polymer particles characterization is presented. Fig. 1b shows SEM micrograph of such powder. It can be observed that particles are between $1 \mu \mathrm{m}$ and $110 \mu \mathrm{m}$, with an average size of $48 \mu \mathrm{m}$. Moreover, the particles possess an irregular and rough morphology. The micrograph of Fig. 1b confirms the results of particle size range distribution by LS of Fig. 1a. This polymeric material was chosen in order to have a good polymeric matrix which can plastically deform during the spraying tests by LP-CGS. Moreover, the particle shape of this specific polymer is very helpful for the final coatings roughness and subsequently for final applications, such as gas or liquid contact, self-cleaning or photocatalysis. In this second part, the most important utilised material was characterized; titanium dioxide $\mathrm{TiO}_{2}$ powder was chosen as nanoceramic material for this experimental investigation. As well-know, this n-type semiconductor material has three different crystalline structures: anatase, rutile and brookite. From the engineering point of view, the most interesting $\mathrm{TiO}_{2}$ structures are anatase and rutile. In this work, anatase $\mathrm{TiO}_{2}$ was selected mainly because of its interesting photocatalytic properties that can break down many organic compounds under UV or VIS light $[2,20]$. Photocatalytic properties of anatase titanium dioxide were discovered by Akira Fujishima in 1967 and published in 1972 [29]. Among many photocatalysts materials, $\mathrm{TiO}_{2}$ was the most widely studied and used in many applications because of its strong oxidizing abilities for the decomposition of organic pollutants, chemical stability, long durability, nontoxicity, low cost and transparency to visible light. These photocatalytic characteristics come from the formation of photogenerated charge carriers, i.e. holes and electrons, which occur upon the absorption of ultraviolet (UV) light corresponding to the band gap [2,27]. In Fig. 4a it is possible to observe the sub-micrometric particle size distribution of this starting powder and in Fig. $4 \mathrm{~b}$ it is shown a SEM micrograph as well. X-ray diffraction was also chosen as characterization technique to confirm the $100 \%$ anatase crystalline phase of this raw material (Fig. 5).

\subsection{Milling results and coatings}

Results of AM and CM at different milling times, $30 \mathrm{~min}, 1 \mathrm{~h}$ and $4 \mathrm{~h}$ and with the two different sieved portions are presented. Table 2 with all sample references is presented below:

In Fig. 6 polymeric particles are shown. From the left to the right it is possible to observe particles that were sieved and afterwards the different particles which underwent Attrition Milling process. 
Table 2

References of milled powders.

\begin{tabular}{|c|c|c|c|}
\hline Reference & Powder type & Reference & Powder type \\
\hline PR101-S1 & Sieved $20-40 \mu \mathrm{m}$ & PR101-S11 & Sieved $20-40 \mu \mathrm{m}+\mathrm{CM} 4 \mathrm{~h}$ \\
\hline PR101-S2 & Sieved $60-80 \mu \mathrm{m}$ & PR101-S12 & Sieved $60-80 \mu \mathrm{m}+\mathrm{CM} 30^{\prime}$ \\
\hline PR101-S3 & Sieved $20-40 \mu \mathrm{m}+\mathrm{AM} 30^{\prime}$ & PR101-S13 & Sieved $60-80 \mu \mathrm{m}+\mathrm{CM} 1 \mathrm{~h}$ \\
\hline PR101-S4 & Sieved $20-40 \mu \mathrm{m}+\mathrm{AM} 1 \mathrm{~h}$ & PR101-S14 & Sieved $60-80 \mu \mathrm{m}+\mathrm{CM} 4 \mathrm{~h}$ \\
\hline PR101-S5 & Sieved $20-40 \mu \mathrm{m}+\mathrm{AM} 4 \mathrm{~h}$ & PR101-T1 & Sieved $60-80 \mu \mathrm{m}+\mathrm{TiO}_{2} \mathrm{AM} 30^{\prime}$ \\
\hline PR101-S6 & Sieved $60-80 \mu \mathrm{m}+\mathrm{AM} 30^{\prime}$ & PR101-T2 & Sieved $60-80 \mu \mathrm{m}+\mathrm{TiO}_{2}$ AM $1 \mathrm{~h}$ \\
\hline PR101-S7 & Sieved $60-80 \mu \mathrm{m}+\mathrm{AM} 1 \mathrm{~h}$ & PR101-T3 & Sieved $60-80 \mu \mathrm{m}+\mathrm{TiO}_{2}$ AM $4 \mathrm{~h}$ \\
\hline PR101-S8 & Sieved $60-80 \mu \mathrm{m}+\mathrm{AM} 4 \mathrm{~h}$ & PR101-T4 & Sieved $60-80 \mu \mathrm{m}+\mathrm{TiO}_{2} \mathrm{CM} 30^{\prime}$ \\
\hline PR101-S9 & Sieved $20-40 \mu \mathrm{m}+\mathrm{CM} 30^{\prime}$ & PR101-T5 & Sieved $60-80 \mu \mathrm{m}+\mathrm{TiO}_{2} \mathrm{CM} 1 \mathrm{~h}$ \\
\hline PR101-S10 & Sieved $20-40 \mu \mathrm{m}+\mathrm{CM} 1 \mathrm{~h}$ & PR101-T6 & Sieved $60-80 \mu \mathrm{m}+\mathrm{TiO}_{2} \mathrm{CM} 4 \mathrm{~h}$ \\
\hline
\end{tabular}
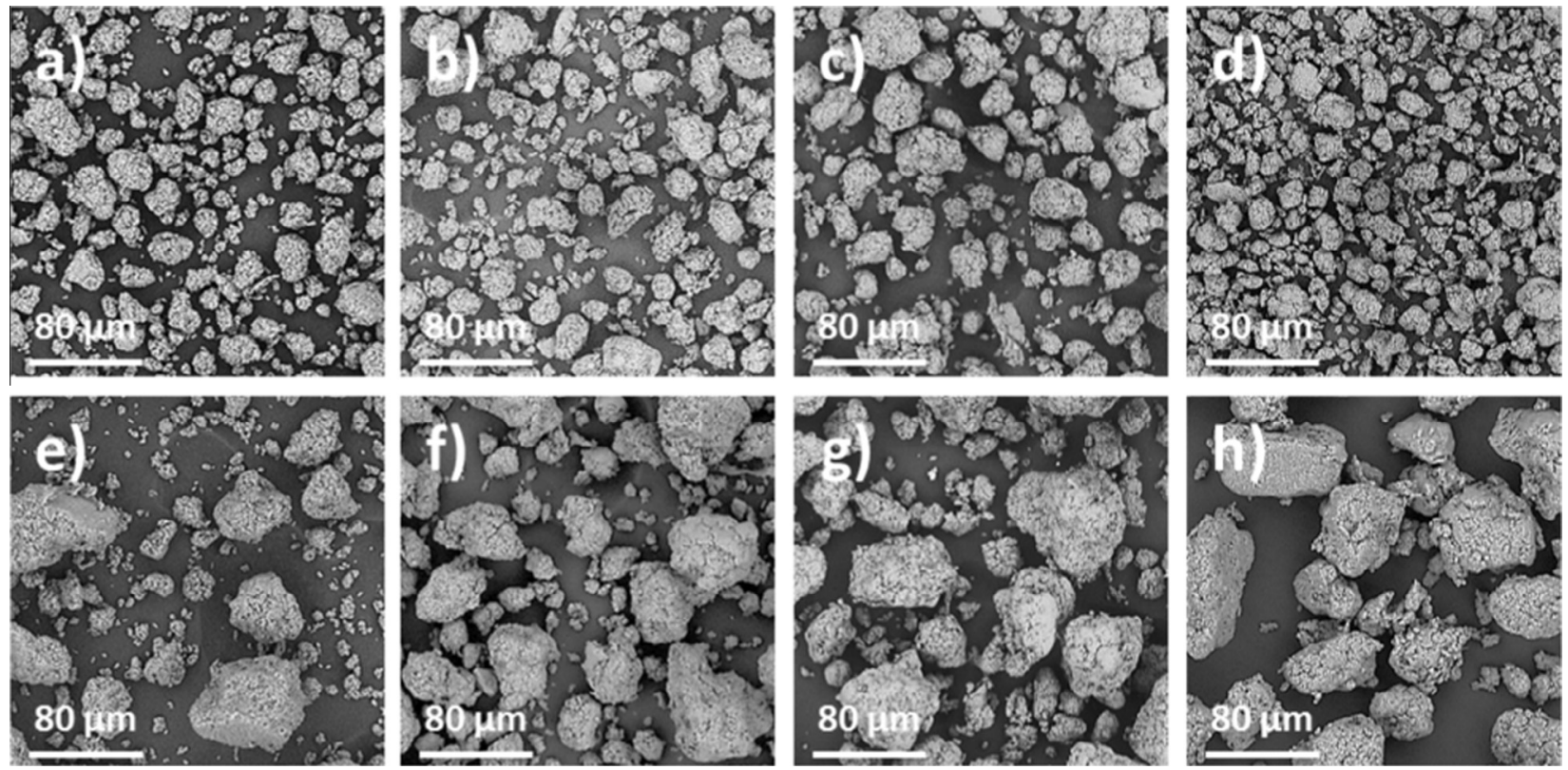

Fig. 6. AM micrographs of the sieved polymer at different times. (a) PR101-S1; (b) PR101-S3; (c) PR101-S4; (d) PR101-S5; (e) PR101-S2; (f) PR101-S6; (g) PR101-S7; (h) PR101-S8.
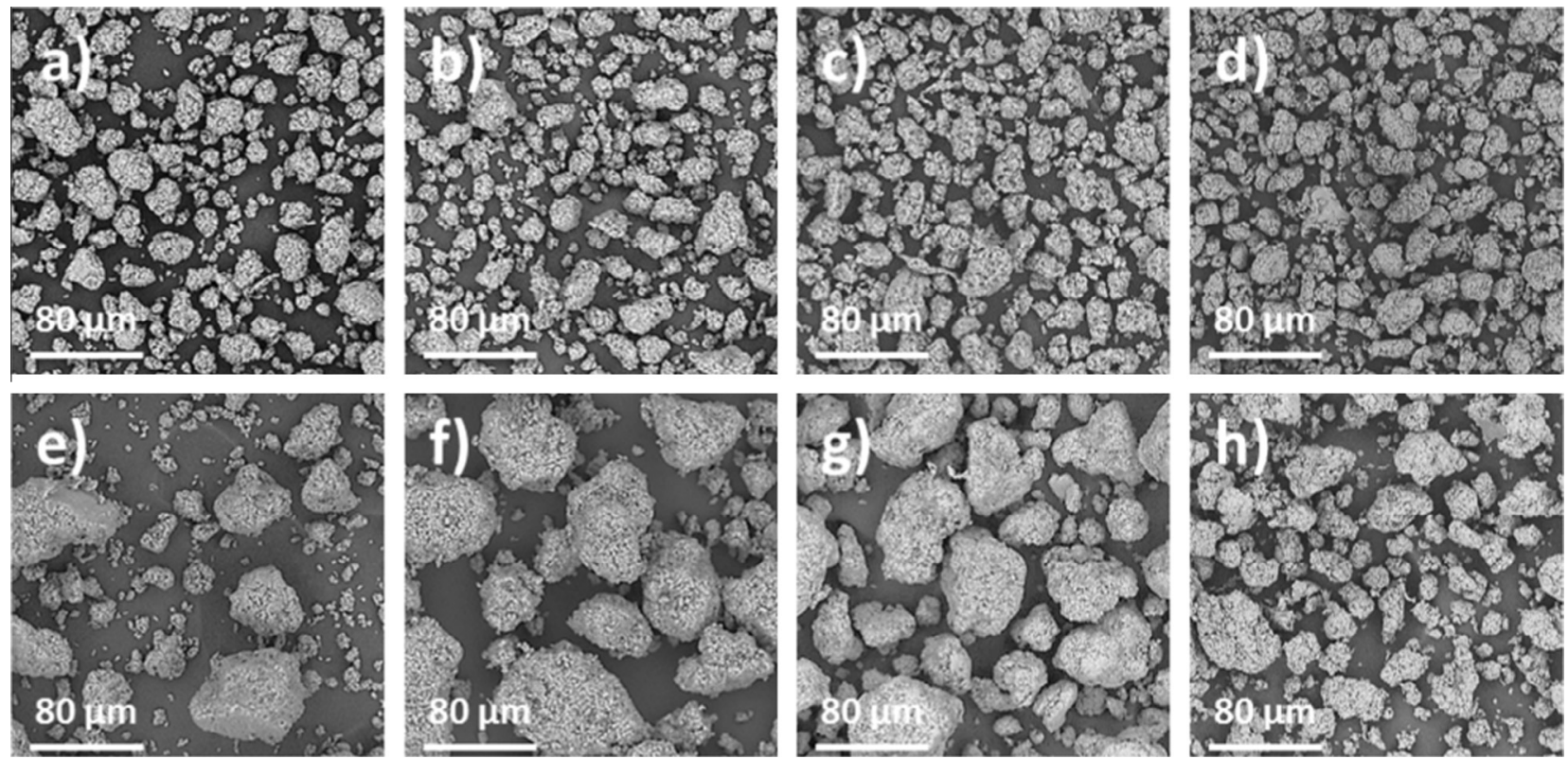

Fig. 7. CM micrographs of the sieved polymer at different times. (a) PR101-S1; (b) PR101-S9; (c) PR101-S10; (d) PR101-S11; (e) PR101-S2; (f) PR101-S12; (g) PR101-S13; (h) PR101-S14.

Please cite this article in press as: M. Robotti et al., Attrition and Cryogenic milling powder production for Low Pressure Cold Gas Spray and composite coatings characterization, Advanced Powder Technology (2016), http://dx.doi.org/10.1016/j.apt.2016.04.014 
In these micrographs it is possible to notice the first effects of milling times. Polymeric particles sieved between 20 and $40 \mu \mathrm{m}$ firstly present phenomena of union and finally after $4 \mathrm{~h}$ of AM they undergo milling process and their dimensions decrease. Regarding polymeric powder sieved $60-80 \mu \mathrm{m}$, particle surfaces start to deform and be more rounded; it is possible to confirm also the reduction in particle size distribution after the longest mixing time. In the subsequent Fig. 7 results of the cryomilled polymer are presented. The superficial embrittlement of particles due to low temperature causes a higher decrease in particle size distribution. The difference between AM and CM is clear: CM system is capable to decrease more particles dimensions due to the lower temperature given by liquid $\mathrm{N}_{2}$ inside the milling environment $[3,4]$. The particle shapes are also influenced by the different equipments and by the temperature below the brittle-ductile transition [13]. Usually, $\mathrm{CM}$ offers more breaking of particles, while AM presents more plastic deformation of the surfaces. In the case of $20-40 \mu \mathrm{m}$ sieved particles, the energy transferred by the crashes between balls, particles and vessels are lower. Results from micrographs 6 and 7 , together with the purpose of the work, have led to the choice to mechanically mix with $\mathrm{TiO}_{2}$ nanoparticles only the $60-80 \mu \mathrm{m}$ distributions. In fact, with this specific particle size distribution $60-80$, the superficial plastic deformation of the polymeric particles is higher compared with the second studied range $20-40$. So, the following mechanical union with $\mathrm{TiO}_{2}$ nanoparticles can be favoured. Fig. 8 shows these new composite powders which can be sprayed by LP-CGS process [18-20]. It is possible to notice that after $30 \mathrm{~min}$ (Fig. 8a) only few titania particles are well mechanically mixed with the polymer. Therefore, this mixing time was not enough to anchor both particles and it was increased up to $4 \mathrm{~h}$. In this other case (Fig. 8c), the particles size reduction was too high, particles were too much deformed and the initial morphology quite rounded and adequate for LP-CGS process was lost. One the other hand, it is possible to observe that after $1 \mathrm{~h}$ of AM mixing (Fig. 8b), $\mathrm{TiO}_{2}$ nanoparticles are very well attached to the

Table 3

LP-CGS spraying conditions.

\begin{tabular}{lll}
\hline Spraying distance $(\mathrm{mm})$ & Gun velocity $(\mathrm{mm} / \mathrm{s})$ & Temperature $\left({ }^{\circ} \mathrm{C}\right)$ \\
\hline $10-50$ & $100-500$ & $50-500$ \\
\hline
\end{tabular}
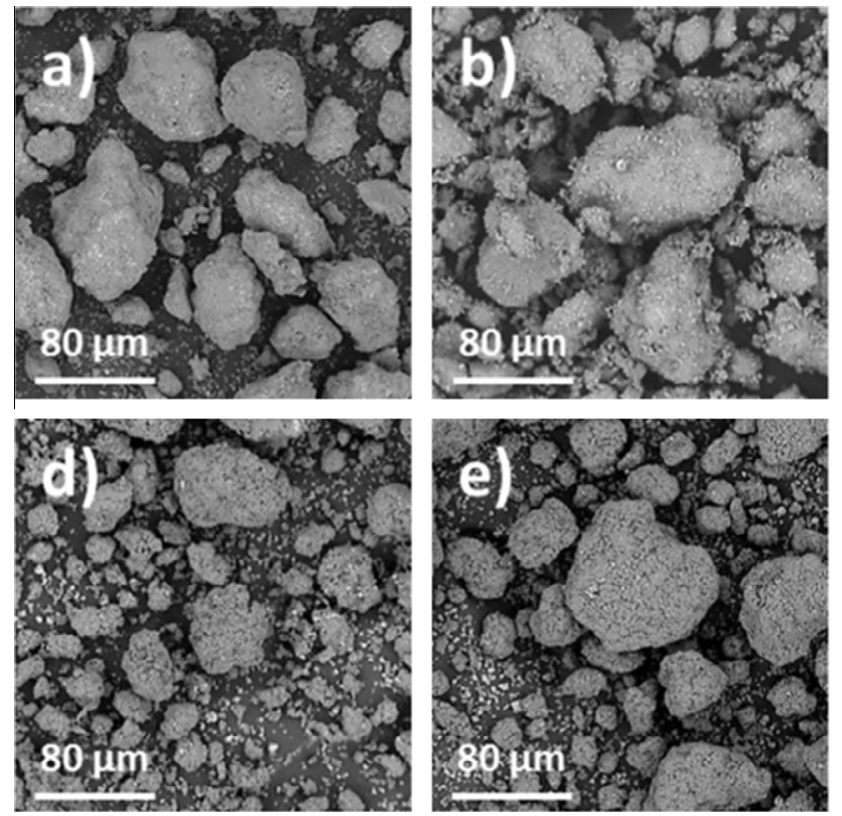
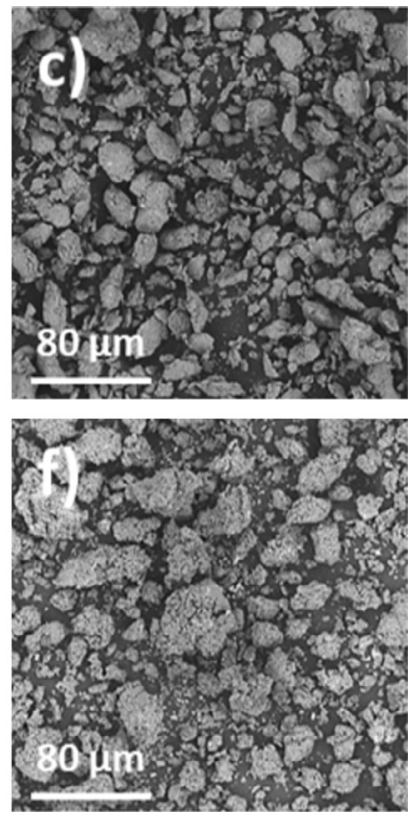

Fig. 8. Composite mixtures at various blending times by AM and CM. (a) PR101-T1; (b) PR101-T2; (c) PR101-T3; (d) PR101-T4; (e) PR101-T5; (f) PR101-T6.
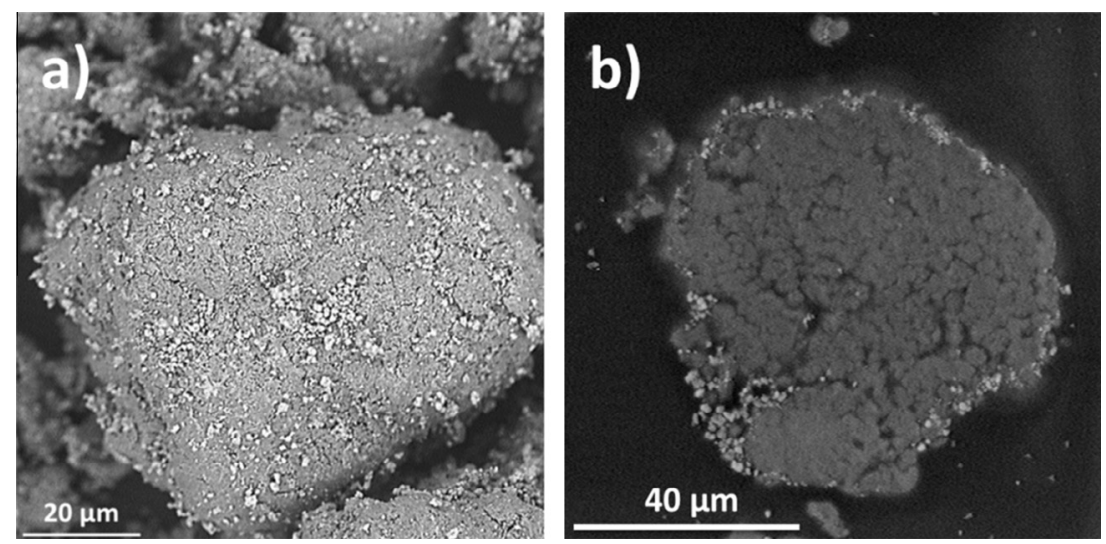

Fig. 9. SEM top surface micrograph (a) and SEM cross section micrograph (b) of PR101-T2 composite powder at higher magnifications. 

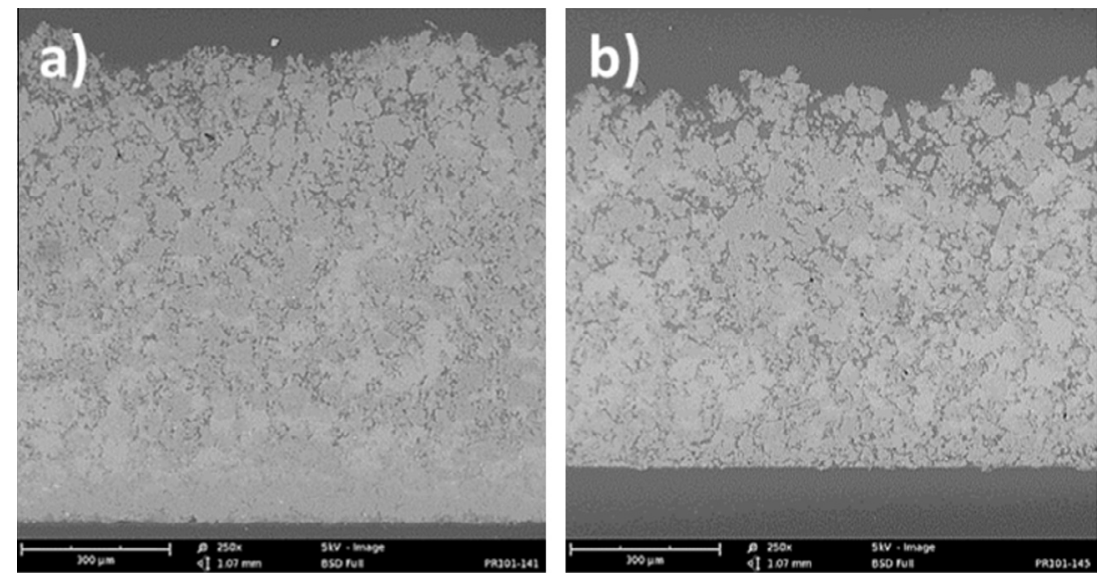

Fig. 10. SEM micrographs of coatings obtained by PR101-T2 (a) and PR101-T5 (b) blends.

polymeric surfaces and homogeneously distributed as well. For all these reasons, the optimal blending time by AM has been selected as 1 hour. This result has the same trend of a previous CPT study [2] in which the best composite mixture obtained by Low Energy Ball Milling method was achieved after $1 \mathrm{~h}$ of milling mechanism. The optimal composite powder, named PR101-T2, was compared with the same of $1 \mathrm{~h}$ by CM, PR101-T5. The difference is clear: in the second case it is present a higher embrittlement effect and this factor leads to a worse mechanical anchoring of titanium dioxide submicrometric particles onto the polymeric ones. In Fig. 9 it is possible to observe a micrograph at higher magnification of the best mechanically mixed composite powder, made from 60 to $80 \mu \mathrm{m}$ sieved polymer and $\mathrm{TiO}_{2}$ articles mixed for $1 \mathrm{~h}$. In this case, particles are sufficiently deformed on the surface to let the ceramic nanoparticles be very well mechanically anchored. This powder presents also more rounded shape, which will be a good factor for LP-CGS spraying tests. Regarding CM process, the best mixture was also considered the one of $1 \mathrm{~h}$ because $30 \mathrm{~min}$ of mechanical milling are not enough for having a well anchoring and $4 \mathrm{~h}$ are too much because first particles deformation processes appear. For these reasons, powders PR101-T2 and PR101-T5 were the ones which were selected to be sprayed for the first time by Low Pressure Cold Gas Spray. Spraying conditions had to be found, good compromises between temperature, pressure and distances must be discovered and also good deposition parameters for the composite mixtures. The following Table 3 shows the specific parameters used for spraying these novel composite powders:

Once all these technical parameters were controlled and good deposition efficiency was obtained onto both stainless steel and polymeric substrates. The characterization presented in this work regards the second type of substrate, PS polystyrene polymer. The characterization of coatings cross sections is presented, while a second deeper investigation of mechanical properties and functional properties started to be evaluated. In Fig. 10a first coatings obtained with PR101-T2 powder are presented. On the other hand, in Fig. 10b a SEM micrograph of a coating manufactured with cryomilled PR101-T5 feedstock is shown as well. Fundamental coatings parameters such as thickness, porosity and superficial roughness were determined; they are shown in Table 4 below, together with the corresponding standard deviations:

Table 4

Coatings parameters.

\begin{tabular}{llll}
\hline & Thickness $(\mu \mathrm{m})$ & Porosity $(\%)$ & Roughness Ra $(\mu \mathrm{m})$ \\
\hline PR101-T2 & $957.1 \pm 20.8$ & $12.0 \pm 2.2$ & $17.0 \pm 1.4$ \\
PR101-T5 & $768.6 \pm 19.3$ & $9.8 \pm 2.2$ & $14.0 \pm 2.6$
\end{tabular}

It is possible to notice that $\mathrm{CM}$ coatings present lower thickness, porosity and roughness compared with AM ones. Considering that these powders possess the same particle size distribution after sieving $(60-80 \mu \mathrm{m})$, the reason of this difference is related to the higher deformation and compacting of the material during the milling process. More dense and compacted starting particles give lower thickness and less porosity. Moreover, inside LP-CGS spraying process, particle dimensions are strictly related to the superficial roughness and subsequently final Ra values of CM coatings are lower too.

\section{Conclusions}

- Different ceramic-polymer composite powders were obtained and successively sprayed by Low Pressure Cold Gas Spray technique.

- The anchoring mechanism between polymer microparticles and ceramic nanoparticles during two different mixing processes such as Attrition and Cryogenic Milling was studied and explained. It was found that the mechanical anchoring of Attrition Milling was more efficient due to the plastic deformation of the surface of the polymeric particles. The behaviour of the polymer is similar to a rigid solid because the milling process temperature is below its $\mathrm{Tg}$. This fact led to embrittlement effect, higher reduction of particle size and finally lower efficiency in the mechanical anchoring between polymeric particles and ceramic ones.

- The characterization of the different obtained composite powders was carried out. SEM, LS and XRD were fundamental characterization instruments to observe evolution and changes of the particles, especially during the mixing processes.

- The selected composite mixture is the one obtained sieving polymeric particles in the range $60-80 \mu \mathrm{m}$ and milling them with $\mathrm{TiO}_{2}$ inside Attrition Mill for $1 \mathrm{~h} .30 \mathrm{~min}$ of milling time were not enough to achieve a proper anchoring among particles and $4 \mathrm{~h}$ are too much because of the increased number of fractured particles. Changes in particle size distribution and in shape were observed, while titania anatase crystalline phase was preserved.

- This well mechanical-bonded composite mixture which was later sprayed by Low Pressure Cold Gas Spray technology. First coatings were obtained, taking into account mechanical adhesion and CTE coefficient with the substrate, thickness, porosity and superficial roughness. Furthermore, these novel types of $\mathrm{TiO}_{2}$ composite coatings can offer a perfect base for their applicability as photocatalysts materials. 


\section{Acknowledgements}

The authors wish to thank Generalitat de Catalunya (2014 SGR1558) and Ministerio de Economía y Competitividad del Gobierno de España (MAT2013 46755R) for the financial support for this research project.

\section{References}

[1] R. Quesada-Cabrera, A. Mills, C. O'Rourke, Action spectra of P25 $\mathrm{TiO}_{2}$ and a visible light absorbing, carbon-modified titania in the photocatalytic degradation of stearic acid, Appl. Catal. B 150-151 (2014) 338-344.

[2] M. Robotti, M. Gardon, S. Dosta, A. Concustell, I.G. Cano, J.M. Guilemany, Selfcleaning, photocatalytic and superhydrophobic coatings obtained by Cold Gas Spray, Intell. Prop. Prot. IPP (2014).

[3] C. Suryanarayana, Mechanical alloying and milling, Prog. Mater Sci. 46 (2001) $1-184$.

[4] P.J. Hubert, K. Kathiresan, K. Wakabayashi, Filler exfoliation and dispersion in polypropylene/as-received graphite nanocomposites via cryogenic milling, Polym. Sci. (2011) 2275.

[5] D.B. Witkin, EJ Lavernia, Synthesis and mechanical behaviour of nanostructured materials via cryomilling, Prog. Mater Sci. (2006) 1-60.

[6] G. Zhang, A.K. Scharb, S. Tria, O. Elkedim, Tensile and tribological behaviors of PEEK/nano- $\mathrm{SiO}_{2}$ composites compounded using a ball milling technique, Compos. Sci. Technol. 68 (2008) 3073-3080.

[7] M. Senna, V. Šepelák, J. Shi, B. Bauer, A. Feldhoff, V. Laporte, K.D. Becker, Introduction of oxygen vacancies and fluorine into $\mathrm{TiO}_{2}$ nanoparticles by comilling with PTFE, J. Solid State Chem. 187 (2012) 51-57.

[8] D. Olmos, C. Domínguez, P.D. Castrillo, J. Gonzalez-Benito, Crystallization and final morphology of HDPE: effect of the high energy ball milling and the presence of $\mathrm{TiO}_{2}$ nanoparticles, Polymer 50 (2009) 1732-1742.

[9] J. González-Benito, G. González-Gaitano, Interfacial conformations and molecular structure of PMMA in PMMA/silica nanocomposites. Effect of high-energy ball milling, Macromolecule 41 (2008) 4777-4785.

[10] D. Olmos, E. Rodríguez-Gutiérrez, J. González-Benito, Polymer Structure and Morphology of Low Density Polyethylene Filled With Silica Nanoparticles, Wiley Online Library (2012).

[11] W. Mekprasart, W. Jarenboon, W. Pecharapa, $\mathrm{TiO}_{2} / \mathrm{CuPc}$ hybrid nanocomposites prepared by low-energy ball milling for dye-sensitized solar cell application, Mater. Sci. Eng., B 172 (2010) 231-236.

[12] R. Amade, P. Heitjans, S. Indris, M. Finger, A. Haeger, D. Hesse, Defect formation during high-energy ball milling in $\mathrm{TiO}_{2}$ and its relation to the photocatalytic activity, J. Photobiol. A: Chem. 207 (2009) 231-235.

[13] Y.G. Zhu, Z.Q. Li, D. Zhang, T. Tanimoto, PET/SiO 2 nanocomposites prepared by cryomilling, J. Polym. Sci., Part B: Polym. Phys. 44 (2006) 1161-1167.

[14] M. Fabián, G. Tyuliev, A. Feldhoff, N. Kostova, P. Kollár, S. Suzuki, F. Saito, V. Šepelák, One-step synthesis of nanocrystalline ZnO via cryomilling, Powder Technol. 235 (2013) 395-399.
[15] S. Amirkhanlou, M. Ketabchi, N. Parvin, Nanocrystalline/nanoparticle ZnO synthesized by high energy ball milling process, Mater. Lett. 86 (2012) 122 124.

[16] R.K. Sendi, S. Mahmud, Quantum size effect on ZnO nanoparticle-based discs synthesized by mechanical milling, Appl. Surf. 258 (2012) 8026-8031.

[17] D. Ma, Y.A. Akpalu, Y. Li, R.W. Siegel, L.S. Schadler, Effect of titania nanoparticles on the morphology of low density polyethylene, J. Polym. Sci., Part B: Polym. Phys. 43 (2005) 488-497.

[18] D.M. Chun, S.H. Ahn, Deposition mechanism of dry sprayed ceramic particles at room temperature using a nano-particle deposition system, Acta Mater. (2011) 2693-2703.

[19] J.B. Powers, Method for flame spraying polyethylene, 1955. Publication number: US2718473 A.

[20] M. Yamada, H. Isago, H. Nakano, M. Fukumoto, Cold spraying of $\mathrm{TiO}_{2}$ photocatalyst coating with nitrogen process gas, J. Therm. Spray Technol. 19 (2010) 1218-1223.

[21] G. Bae, Y. Xiong, S. Kumar, K. Kang, C. Lee, General aspects of interface bonding in kinetic sprayed coatings, Acta Mater. 56 (2008) 4858-4868.

[22] E. Leivo, T. Wilenius, T. Kinos, P. Vuoristo, T. Mäntylä, Properties of thermally sprayed fluoropolymer PVDF, ECTFE, PFA and FEP coatings, Prog. Org. Coat. 49 (2004) 69-73.

[23] C. Mateus, S. Costil, R. Bolot, C. Coddet, Ceramic/fluoropolymer composite coatings by thermal spraying - a modification of surface properties, Surf. Coat. Technol. 191 (2005) 108-118.

[24] A.S. Alhulaifi, G.A. Buck, W.J. Arbegast, Numerical and experimental investigation of cold spray gas dynamic effects for polymer coating, J. Therm. Spray Technol. 21 (2012) 852-862.

[25] A. Moridi, S.M. Hassani-Gangaraj, M. Guagliano, M. Dao, Cold spray coating: review of material systems and future perspectives, Surf. Eng. 36 (2014) 369395.

[26] Y. Xu, I.M. Hutchings, Cold spray deposition of thermoplastic powder, Surf. Coat. Technol. 201 (2006) 3044-3050.

[27] M. Gardon, C. Fernández-Rodríguez, D. Garzón Sousa, J.M. Doña-Rodríguez, S. Dosta, I.G. Cano, J.M. Guilemany, Photocatalytic activity of nanostructured anatase coatings obtained by cold gas spray, J. Therm. Spray Technol. 23 (2014) 1135-1141.

[28] M. Yamada, H. Isago, K. Shima, H. Nakano, M. Fukumoto. Deposition of $\mathrm{TiO}_{2}$ Ceramic Particles on Cold Spray Process. International Thermal Spray Conference ITSC 2010, Proceedings.

[29] A. Fujishima, K. Honda, Electrochemical photolysis of water at a semiconductor electrode, Nature 238 (1972) 37-38.

[30] J.M. Guilemany, N. Cinca, L. Casas, E. Molins, Ordering and disordering processes in MA and MM intermetallic iron aluminade powders, J. Mater. Sci. 44 (2009) 2152-2161.

[31] N. Cinca, E. Hurtado, I.G. Cano, J.M. Guilemany, Mechanical milling of a nanostructured ductile iron powder under dry, wet and cryogenic atmospheres, Revista de Metalurgia 47 (2011) 197-204. 